\title{
Evaluation of the BioMérieux VIDAS® HIV Duo Quick and Anti-HCV Assays for Dried Blood Spot Based Serosurveillance
}

\section{François Cholette ( $D$ Francois.Cholette@phac-aspc.gc.ca)}

National HIV and Retrovirology Laboratories, National Microbiology Laboratory at the J.C. Wilt Infectious Diseases Research Centre, Public Health Agency of Canada

Braedy Farmer

National HIV and Retrovirology Laboratories, National Microbiology Laboratory at the J.C. Wilt Infectious Diseases Research Centre, Public Health Agency of Canada

\section{Olga Balakireva}

Institute for Economics and Forecasting, Ukrainian National Academy of Sciences

\section{Daria Pavlova}

Ukrainian Institute for Social Research After Oleksandr Yaremenko

\section{Anna Lopatenko}

Dnipropetrovsk Oblast Medical Centre of Socially Significant Diseases

Iryna Chukhalova

Dnipropetrovsk Oblast Medical Centre of Socially Significant Diseases

\section{Svitlana Bargan}

Dnipropetrovsk Oblast Medical Centre of Socially Significant Diseases

\section{Sharmistha Mishra}

MAP Centre for Urban Health Solutions, Li Ka Shing Knowledge Institute, St. Michael's Hospital

\section{Marissa Becker}

Institute for Global Public Health, Department of Community Health Sciences, Rady Faculty of Health

Sciences, University of Manitoba

\section{Emma R. Lee}

National HIV and Retrovirology Laboratories, National Microbiology Laboratory at the J.C. Wilt Infectious Diseases Research Centre, Public Health Agency of Canada

\section{John Kim}

National HIV and Retrovirology Laboratories, National Microbiology Laboratory at the J.C. Wilt Infectious Diseases Research Centre, Public Health Agency of Canada

\section{Paul Sandstrom}

National HIV and Retrovirology Laboratories, National Microbiology Laboratory at the J.C. Wilt Infectious Diseases Research Centre, Public Health Agency of Canada 


\section{Research Article}

\section{Keywords:}

Posted Date: February 21st, 2022

DOI: https://doi.org/10.21203/rs.3.rs-1350673/v1

License: (c) (i) This work is licensed under a Creative Commons Attribution 4.0 International License. Read Full License 


\section{Abstract}

Serosurveillance is central to monitoring our progress towards HIV and HCV elimination targets proposed for 2030. However, serosurveillance systems are ineffective without reliable serological assays for the detection of HIV and HCV antibodies. Assays should also be compatible with dried blood spot (DBS) samples to facilitate biological sample collection. The VIDAS ${ }^{\circledR}$ HIV Duo Quick and Anti-HCV assays are sold as reagents strips and processed by the automated VIDAS ${ }^{\circledR}$ benchtop immunoanalyser. While both assays have shown excellent performance in serum and plasma, performance data in DBS samples is lacking. In our study, we evaluate the performance of the VIDAS ${ }^{\circledR}$ HIV Duo Quick and Anti-HCV assays in DBS $(n=725)$ collected during a cross-sectional serosurvey (the Transitions study). The VIDAS ${ }^{\circ}$ HIV Duo quick had a sensitivity and specificity of $94.5 \%(95 \% \mathrm{Cl}: 85.1 \%, 98.5 \%)$ and $95.7 \%$ (95\% Cl: $93.9 \%, 97.0 \%)$ respectively. Likewise, the VIDAS® Anti-HCV had a sensitivity and specificity of $95.6 \%$ (95\% Cl: $91.6 \%$, $97.8 \%)$ and $95.6 \%(95 \% \mathrm{Cl}$ : 93.5\%, 97.0\%) respectively. These assays are unlikely to be helpful in lowprevalence settings due to sub-optimal performance, but their performance could likely be improved by optimizing DBS elution protocols which was, unfortunately, not possible during our study.

\section{Introduction}

Human immunodeficiency virus (HIV) and hepatitis C virus (HCV) infections remain significant public health concerns globally ${ }^{1,2}$. Roughly 1.5 million people became newly infected with HIV and HCV in 2020 according to estimates from the Joint United Nations Programme on HIV/AIDS (UNAIDS) ${ }^{3}$ and the World Health Organization (WHO) ${ }^{1}$ respectively. The prevalence of HIV and HCV co-infection among key populations is also concerning, especially among people who inject drugs (PWID) $)^{4,5}$. Aided by highly active antiretroviral therapy (ART) and direct-acting antivirals (DAAs), ambitious targets for the elimination of HIV and HCV as public health threats have been set for 20301,6. The UNAIDS 95-95-95 targets stipulate that $95 \%$ of people living with HIV (PLWH) should be aware of their HIV status, $95 \%$ of people who are aware of their status should be receiving treatment, and $95 \%$ of people on HIV treatment should be virally suppressed ${ }^{6}$. Along a similar vein, the WHO suggest we aim to treat $80 \%$ of $\mathrm{HCV}$ infections among those eligible, reduce the incidence of new HCV infections by $90 \%$, and reduce liverrelated mortality by $65 \%{ }^{1}$. Progress towards these elimination targets can be monitored by behavioural and biological serosurveillance systems but, they necessitate accurate serological assays for the detection of HIV and HCV antibodies ${ }^{7}$. Serological assays should also be compatible with dried blood spot (DBS) samples to facilitate the implementation of surveillance by reducing structural and geographical barriers to biological specimen collection $^{8,9}$.

The VIDAS ${ }^{\circledR}$ HIV Duo Quick (bioMérieux, Marcy-l'Étoile, France) is a 4th generation enzyme-linked fluorescent assay (ELFA) capable of detecting the p24 antigen and antibodies to HIV ${ }^{10}$. The VIDAS ${ }^{8}$ Anti-HCV is a 3rd generation ELFA that makes use of synthetic core, NS3, NS4A, and NS4B antigens to detect antibodies to $\mathrm{HCV}^{11}$. Both assays are sold as ready-to-use reagents strips containing all necessary reagents and protocols (i.e., washing, incubation, and detection) are automated by the VIDAS ${ }^{\circ}$ 
benchtop immunoanalyser thereby minimising operator error. Although the VIDAS $®$ system is considered low to medium throughput, it merits consideration due to its accessibility. The VIDAS $®$ system is simpler to operate compared to manual, 96-well plate based serological assays and cost significantly less $(\$ 50,000 \mathrm{CDN})$ than other commercial immunoanalysers like the Abbott Alinity or Bio-Rad BioPlex 2200 $\left(\$ 200,000\right.$ to $\$ 300,000$ CDN). The VIDAS ${ }^{\circledR}$ HIV Duo Quick and Anti-HCV have shown excellent performance in serum and plasma but, performance data in DBS are currently lacking especially in the context of serosurveillance ${ }^{10-17}$.

In our study, we evaluate the performance of the VIDAS ${ }^{\circledR}$ HIV Duo Quick and Anti-HCV assay for the detection of HIV and HCV antibodies respectively in DBS for the purpose of serosurveillance. These assays were evaluated against a subset of DBS samples $(n=725)$ collected during a cross-sectional integrated biological and behavioural survey in the city of Dnipro, Ukraine. Sensitivity, specificity, positive predictive values, and negative predictive values were estimated using the Avioq ${ }^{\circledR}$ HIV-1 Microelisa System or Ortho ${ }^{\circledR}$ HCV v3.0 ELISA Test System as the reference test. Both reference tests are central to the Public Health Agency of Canada's behavioural and biological surveillance system that monitors the prevalence of HIV and hepatitis $\mathrm{C}$ among key populations in Canada using DBS samples ${ }^{8}$.

\section{Results}

A random subset of DBS samples $(n=725)$ was chosen from the Transitions study to be tested retrospectively with the VIDAS ${ }^{\circledR}$ HIV Duo Quick and Anti-HCV assays to assess their performance (Table 1). In this subset, the Avioq ${ }^{\circledR}$ HIV-1 Microelisa System and Ortho ${ }^{\circledR}$ v3.0 ELISA Test System were positive in 7.6\% (55/725) and 27.4\% (199/725) DBS samples respectively. The VIDAS $\AA$ HIV Duo Quick was positive in $96.4 \%$ (53/55) and negative in $82.7 \%$ (554/670) of the HIV positive and HIV negative DBS samples respectively corresponding to a sensitivity of $96.4 \%$ (95\% Cl: $87.7 \%, 99.4 \%)$, specificity of $82.7 \%$ (95\% Cl: $79.6 \%, 85.4 \%)$, PPV of $31.4 \%$ (95\% Cl: $24.8 \%, 38.7 \%)$, and NPV of 99.6\% (95\% Cl: $98.7 \%, 99.9 \%$ ). The VIDAS $\circledast$ HIV Duo Quick was in moderate agreement (kappa $=0.405$ [95\% Cl: 0.326, 0.485]) with the Avioq ${ }^{\circledR}$ HIV-1 Microelisa System based on kappa coefficients (Table 1). Figure 1 illustrates that the VIDAS ${ }^{\circledR}$ HIV Duo Quick can adequately discriminate between HIV positive and HIV negative DBS. Furthermore, receiver operating characteristic (ROC) curve analysis suggested that performance could be improved by adjusting the test value cutoff provided by the manufacturer (Fig. 1). Adjusting the test value significantly improved specificity (95.7\% [95\% Cl: 93.9\%, 97.0\%] versus $82.7 \%$ [95\% Cl: 79.6\%, 85.4\%]) and agreement with the Avioq ${ }^{\circledR}$ HIV-1 Microelisa System $(0.741$ [95\% Cl: $0.656,0.826]$ versus 0.405 [95\% Cl: $0.326,0.485])$. 
Table 1

Performance statistics for the detection of HIV and HCV antibodies in DBS samples using the VIDAS ${ }^{\circledR}$ HIV Duo Quick and Anti-HCV.

\begin{tabular}{|c|c|c|c|c|}
\hline \multirow[b]{2}{*}{ Cutoff (test value) } & \multicolumn{2}{|c|}{ VIDAS $®$ HIV Duo Quick } & \multicolumn{2}{|l|}{ VIDAS® Anti-HCV } \\
\hline & $0.25^{a}$ & $0.30^{b}$ & $1.00^{\mathrm{a}}$ & $0.80^{b}$ \\
\hline Sensitivity $(\mathrm{n} / \mathrm{N})$ & $53 / 55$ & $52 / 55$ & $175 / 199$ & $185 / 199$ \\
\hline$\%(95 \% \mathrm{Cl})$ & $96.4(87.7,99.4)$ & $94.5(85.1,98.5)$ & $87.9(82.7,91.8)$ & $93.0(88.5,95.8)$ \\
\hline Specificity $(n / N)$ & $554 / 670$ & $641 / 670$ & $518 / 526$ & $503 / 526$ \\
\hline$\%(95 \% \mathrm{Cl})$ & $82.7(79.6,85.4)$ & $95.7(93.9,97.0)$ & $98.5(97.0,99.2)$ & $95.6(93.5,97.1)$ \\
\hline $\operatorname{PPV}(n / N)$ & $116 / 670$ & $(29 / 670)$ & $8 / 526$ & $23 / 526$ \\
\hline$\%(95 \% \mathrm{Cl})$ & $31.4(24.8,38.7)$ & $64.2(53.3,73.8)$ & $95.6(91.6,0.97 .8)$ & $88.9(84.0,92.5)$ \\
\hline NPV (n/N) & $2 / 55$ & $3 / 55$ & $24 / 199$ & $14 / 199$ \\
\hline$\%(95 \% \mathrm{Cl})$ & $99.6(98.7,99.9)$ & $99.5(98.6,99.9)$ & $95.6(93.5,97.0)$ & $97.3(95.5,98.4)$ \\
\hline Kappa & 0.405 & 0.741 & 0.886 & 0.874 \\
\hline$(95 \% \mathrm{Cl})$ & $(0.326,0.485)$ & $(0.656,0.826)$ & $(0.848,0.925)$ & $(0.834,0.913)$ \\
\hline \multicolumn{5}{|c|}{${ }^{\mathrm{a}}$ Test value recommended by the manufacturer. } \\
\hline
\end{tabular}

The VIDAS ${ }^{\circledR}$ Anti-HCV was positive in $87.9 \%$ (175/199) and negative in $98.5 \%(518 / 526)$ of the HCV positive and HCV negative DBS samples respectively corresponding to a sensitivity of $95.6 \%(95 \% \mathrm{Cl}$ : 91.6\%, 97.8\%), specificity of 95.6\% (95\% Cl: 93.5\%, 97.0\%), PPV of $95.6 \%$ (95\% Cl: $91.6 \%, 97.8 \%)$, and NPV of $95.6 \%(93.5 \%, 97.0 \%)$. The VIDAS $®$ Anti-HCV was in almost perfect agreement with the Ortho $\AA$ HCV v3.0 ELISA Test System based on kappa coefficients (kappa $=0.886$ [95\% Cl: $0.848,0.925]$ ). The VIDAS ${ }^{\circledR}$ Anti-HCV could also adequately discriminate between HCV positive and HCV negative DBS (Fig. 1) however, adjusting the test value cutoff did not significantly impact performance (Table 1).

\section{Discussion}

Serosurveillance will play a central role locally and internationally in monitoring progress made towards HIV and HCV elimination targets set for $2030^{1,6}$. To be effective, serosurveillance systems require accurate serological assays for the detection of HIV and HCV antibodies ${ }^{7}$. While serum and plasma are typically the preferred biological specimen for serosurveillance, collection is not always convenient or possible due to a lack of infrastructure ${ }^{9,18}$, shortages of trained personnel (i.e., phlebotomists) ${ }^{19,20}$, and barriers to accessing collection sites ${ }^{21}$. Serological assays should also perform satisfactorily with DBS 
samples to facilitate biological sample collection. This may lead to more representative sampling and accurate estimates of disease prevalence.

The VIDAS $®$ HIV Duo quick had a sensitivity and specificity of $94.5 \%$ (95\% $\mathrm{Cl}: 85.1 \%, 98.5 \%$ ) and $95.7 \%$ (95\% Cl: $93.9 \%, 97.0 \%)$ respectively. This is considerably lower compared to performance reported by others using DBS. In a study conducted among PLWH on ART in Kinshasa, Democratic Republic of Congo Barquín et al. ${ }^{12}$ reported a sensitivity of $100 \%$ (95\% Cl: $\left.98.6 \%, 100 \%\right)$ and specificity of $100 \%$ (95\% Cl: $96.0 \%, 100 \%)$ for the VIDAS ${ }^{8}$ HIV Duo Quick. Differences in performance between our study and Barquín et al. could be explained by procedural differences. In the Barquín et al. study, blood was collected by venipuncture and spotted onto 903 Protein Saver Cards with a micropipette. The DBS samples in our study were directly spotted using finger-pokes. Serological assays in general may perform better with DBS samples prepared from venous blood versus finger-pokes ${ }^{22}$. Furthermore, Barquín et al. eluted entire DBS spots $(70 \mu \mathrm{L})$ in phosphate buffered saline (PBS) while we eluted a single $6 \mathrm{~mm}$ DBS punch $(\sim 20$ $\mu \mathrm{L}$ ) in PBS containing $0.5 \%$ BSA and $0.05 \%$ Tween 20 . Sample input (e.g., quantity of punches) and choice of elution buffer significantly impact performance ${ }^{23}$. Although our findings suggest that the VIDAS ${ }^{\circledR}$ Duo Quick may have limited use in low prevalence settings due to a PPV of $64.2 \%(95 \% \mathrm{Cl}$ : $53.3 \%, 73.8 \%$ ), optimizing our elution protocol for DBS samples is likely warranted to achieve a better estimate of the assay's performance. This was not possible during our study due to limited sample volume but, future work will consist of improving our elution protocol using DBS samples contrived ${ }^{9}$ from highly characterized reference serum/plasma samples ${ }^{24}$.

In contrast, the VIDAS ${ }^{8}$ Anti-HCV performed better in our laboratory compared to a study by Carrasco et al. ${ }^{13}$ In this study, DBS samples were collected from adult patients attending hospitals in Kinshasa, Democratic Republic of Congo as part of the Observational Kinshasha AIDS Prevention Initiative (OKAPI) project. The authors reported a sensitivity and specificity of $61.5 \%$ (95\% Cl: $31.6 \%, 86.1 \%)$ and $89.4 \%$ (95\% Cl: $81.3 \%, 94.8 \%)$ respectively for the VIDAS ${ }^{\circledR}$ Anti-HCV. Furthermore, their findings would suggest that the VIDAS ${ }^{\circledR}$ Anti-HCV would be of limited use for serosurveillance due to a PPV and NPV of $44.4 \%$ (95\% Cl: $21.5 \%, 69.2 \%)$ and $94.4 \%(95 \% \mathrm{Cl}: 87.4 \%, 98.1 \%)$ respectively. It would be important to note however, that Carrasco et al. lacked a reference test and relied on two positive/indeterminate results among the three index tests under evaluation to establish "true positives". Even though our findings are more encouraging, improving our DBS elution protocol will likely be necessary to improve performance and make use of the VIDAS ${ }^{\circledR}$ Anti-HCV in low-prevalence settings.

In the present study, we evaluated the performance of the VIDAS® HIV Duo Quick and Anti-HCV assay for the detection of HIV and HCV antibodies respectively in DBS samples for the purpose of serosurveillance. These assays are unlikely to be helpful in low-prevalence settings due to less-than-ideal PPV and NPV. However, it is possible that optimizing DBS elution protocols (e.g., quantity of punches, choice of elution buffer) will improve performance but was not possible during our study. Improving our elution protocols will be investigated in future work with the help of highly characterized reference material. 


\section{Methods}

\section{Study participants and sample collection}

The Transitions study is a cross-sectional integrated biological and behavioural survey conceived to measure the prevalence and patterns of HIV risk and vulnerabilities ${ }^{25,26}$. From March to December 2015, field teams in the city of Dnipro, Ukraine administered written informed consent, conducted interviews, and oversaw multiplex rapid testing for HIV, syphilis, and hepatitis B and C (Profitest Combo, New Vision Diagnostics, Haicang Xiamen, China) among study participants. DBS samples were also collected from consenting participants on 903 Protein Saver Cards (Cytiva Whatman, Marlborough, MA) using Microtainer ${ }^{\circledR}$ high flow contact activated lancets (BD, Franklin Lakes, NJ) for HIV and HCV confirmatory serological testing. Confirmatory testing was performed by the National HIV and Retrovirology Laboratories of the Public Health Agency of Canada (Winnipeg, Canada) using the Avioq HIV-1 Microelisa System (Avioq Inc., Durham, NC) and Ortho ${ }^{\circledR}$ HCV v3.0 ELISA Test System (Ortho-Clinical Diagnostics Inc., Raritan, $\mathrm{NJ}$ ). We enrolled 1,818 participants and all participants consented to providing a DBS sample. A subset of DBS samples ( $n=725$ [39.9\%]) were randomly chosen to evaluate the performance of the bioMérieux VIDAS ${ }^{8}$ HIV Duo Quick and Anti-HCV assays with DBS samples.

\section{Avioq ${ }^{\circledR}$ HIV-1 Microelisa System}

The Avioq ${ }^{\circledR}$ HIV-1 Microelisa System (Avioq Inc., Research Triangle Park, NC) was used as the reference test for the qualitative detection of antibodies to HIV. DBS samples were punched $(1 \times 6 \mathrm{~mm})$ using a pneumatic Dried Blood Spot Punch System (Analytical Sales and Services Inc., Flanders, NJ) into $2 \mathrm{~mL}$ 96-well polypropylene plates (ThermoFisher Scientific, Ottawa, Canada) and processed according to instructions provided by the manufacturer. Cutoff values (COV) were calculated as follows: COV $=\mathrm{NCX}+$ 0.270 where NCX represents the mean of the Negative Calibrator (Avioq Inc.) values. A result $<$ COV and $\geq$ COV was interpreted as negative and positive respectively.

\section{Ortho® HCV v3.0 ELISA Test System}

The Ortho ${ }^{\circledR}$ HCV v3.0 ELISA Test System (Ortho-Clinical Diagnostics Inc., Raritan, NJ) was used as the reference test for the qualitative detection of antibodies to HCV. DBS specimens were punched $(1 \mathrm{X} 6$ $\mathrm{mm}$ ) using a pneumatic Dried Blood Spot Punch System (Analytical Sales and Services Inc.) into $2 \mathrm{~mL}$ 96-well polypropylene plates (ThermoFisher Scientific). DBS punches were eluted in $200 \mu \mathrm{L}$ of Specimen Diluent (Ortho-Clinical Diagnostics Inc.) overnight at $4^{\circ} \mathrm{C}$ without agitation. Afterwards, plates were gently agitated $(400 \mathrm{rpm})$ at room temperature for 30 minutes and $150 \mu \mathrm{L}$ of DBS eluate was transferred into HCV Encoded Antigen Coated Microwell Plates (Ortho-Clinical Diagnostics Inc.) containing $50 \mu \mathrm{L}$ of Specimen Diluent (Ortho-Clinical Diagnostics Inc.) and processed according to instructions provided by the manufacturer. COV were calculated as follows: $\mathrm{COV}=\mathrm{NCX}+0.600$ where $\mathrm{NCX}$ represents the mean of the Negative Control (Ortho-Clinical Diagnostics Inc.) values. The upper and lower limit of the grey zone (UGZ and LGZ respectively) were calculated as follows: $U G Z=C O V X 1.5$ and $L G Z=C O V X$ 0.9. A result $<$ $L G Z, \geq L G Z$ to $<U G Z$, and $\geq U G Z$ was interpreted as negative, indeterminate, and positive respectively. 


\section{VIDAS® HIV Duo Quick}

The VIDAS ${ }^{\circledR}$ HIV Duo Quick (bioMérieux) was used as the index test for the qualitative detection of antibodies to HIV. DBS specimens were punched $(1 \times 6 \mathrm{~mm})$ using a pneumatic Dried Blood Spot Punch System (Analytical Sales and Services Inc.) into $2 \mathrm{~mL}$ 96-well polypropylene plates (ThermoFisher Scientific). DBS punches were eluted in $200 \mu \mathrm{L}$ of DPBS pH 7.4 containing $0.5 \%$ BSA and $0.05 \%$ Tween 20 at room temperature for 1 hour with agitation (1000 rpm). Afterwards, all remaining DBS eluate was transferred into HIV6 Strips (bioMérieux) and processed by an automated VIDAS $\circledast$ benchtop immunoanalyzer (bioMérieux). A test value $<0.25$ and $\geq 0.25$ was interpreted as negative and positive respectively unless stated otherwise. Test values (TV) were calculated as follows: TV = relative fluorescent value / background fluorescence value.

\section{VIDAS® Anti-HCV}

The VIDAS ${ }^{\circledR}$ Anti-HCV (bioMérieux) was used as the index test for the qualitative detection of antibodies to HCV. DBS specimens were punched (1 X $6 \mathrm{~mm}$ ) using a pneumatic Dried Blood Spot Punch System (Analytical Sales and Services Inc.) Into $2 \mathrm{~mL}$ 96-well polypropylene plates (ThermoFisher Scientific). DBS punches were eluted in $100 \mu \mathrm{L}$ of DPBS pH 7.4 containing $0.5 \%$ BSA and $0.05 \%$ Tween 20 at room temperature for 1 hour with agitation (1000 rpm). Afterwards, all remaining DBS eluate was transferred into HCV Strips (bioMérieux) and processed by an automated VIDAS ${ }^{\circledR}$ benchtop immunoanalyzer (bioMérieux). A test value $<1.0$ and $\geq 1.0$ was interpreted as negative and positive respectively unless stated otherwise. Test values (TV) were calculated as follows: TV = relative fluorescent value / background fluorescence value.

\section{Statistical analysis}

Continuous data were summarized using the median and interquartile range (IQR). Categorical data were presented using exact numbers and proportions. Sensitivity, specificity, negative predictive values (NPV), and positive predictive values (PPV) were estimated using the Avioq ${ }^{\circledR}$ HIV-1 Microelisa System or Ortho ${ }^{\circledR}$ HCV v3.0 ELISA Test System as the reference test.

The strength of agreement between the index test (VIDAS ${ }^{\circledR}$ HIV Duo Quick or Anti-HCV) and the corresponding reference test was quantified with kappa coefficients (https://www.graphpad.com/quickcalcs/kappa1/?K=2). Kappa coefficients were interpreted as follows: $<0=$ no agreement, $0-0.20=$ slight agreement, $0.21-0.40=$ fair agreement, $0.41-0.60=$ moderate agreement, $0.61-0.80=$ substantial agreement, and 0.81-1.00 $=$ almost perfect agreement ${ }^{27}$.

Cutoff values for the VIDAS ${ }^{\circledR}$ HIV Duo Quick and Anti-HCV assays were determined by using a combination of receiver operating characteristic (ROC) curves and Youden's $\mathrm{J}$ statistic ${ }^{28}$. Youden's $\mathrm{J}$ statistic was computed as follows: $\mathrm{J}$ = sensitivity + specificity - 1. Prism v9.0.0 (GraphPad Software, San Diego, CA) was used for all data analysis and visualisation. 


\section{Declarations}

\section{Acknowledgments}

We thank the Transitionsstudy participants for generously giving their time to the study. We thank the field teams for their hard work and dedication. We also thank the invaluable contributions from Eve Cheuk, Lyle McKinnon, Robert Lorway, Sevgi Aral, Stephen Moses, Parinita Bhattacharjee,Huiting Ma, Shajylsac, James Blanchard, Christina Daniuk, and Michael Picklesfrom the Transitionsstudy team. This study was made possible thanks to the support of our country partners in Ukraine: Alliance for Public Health, DEF Group, Dnipropetrovsk Oblast AIDS Center, Public Health Center of the Ministry of Health, Ukrainian Institute for Social Research after Oleksandr Yaremenko and CharitableFoundationVirtus. SM is supported by a Tier 2 Canada Research Chair in Mathematical Modeling. The Transitions study was funded by the Canadian Institutes of Health Research (CIHR MOP-13044).

\section{Author contributions}

F.C. conceived and designed the experiments, performed data analysis, curated data, and wrote the initial draft. B.F. performed data analysis, validated laboratory protocols, collected data, and contributed to writing the initial draft. O.B., D.P., A.L., I.C., S.B., S.M., and M.B.managed, coordinated, and executed several aspects of the Transitions study including biological specimen collection. They also made contributions to the acquisition of financial support for the Transition study leading to this publication. S.M., M.B., E.R.L., J.K., and P.S.provided supervision and critical review of the initial draft.

\section{Competing interests}

All authors declare no potential conflict of interest.

\section{Data availability}

All data generated or analysed during this study are included in this published article (and its Supplementary Information files).

\section{Ethics}

The Transition study was reviewed and approved by the Human Research Ethics Board at the University of Manitoba in Canada (HS16557 [H2013:295]), the Ethical Review Committee Board at the Sociological Association of Ukraine, Committee on Medical Ethics of the L. Gromashevsky Institute of Epidemiology and Infectious Diseases at the National Academy of Medical Sciences of Ukraine. All experiments were carried out in accordance with relevant guidelines and regulations. All participants provided written informed consent. Participants under 16 years of age were considered mature minors and provided consent to take part in the study without consent from their parent or guardian. Written informed consent from the participant's legal guardian/next of kin was not required to participate in this study in accordance with the local legislation and the Human Research Ethics Board at the University of Manitoba 
in Canada and the Ethical Review Committee Board at the Sociological Association of Ukraine, Committee on Medical Ethics of the L. Gromashevsky Institute of Epidemiology and Infectious Diseases at the National Academy of Medical Sciences of Ukraine.

\section{References}

1 Dore, G. J. \& Bajis, S. Hepatitis C virus elimination: laying the foundation for achieving 2030 targets. Nature Reviews Gastroenterology \& Hepatology18, 91-92, doi:10.1038/s41575-020-00392-3 (2021).

2 Pandey, A. \& Galvani, A. P. The global burden of HIV and prospects for control. The Lancet HIV6, e809e811, doi:10.1016/s2352-3018(19)30230-9 (2019).

3 HIV/AIDS. UNAIDS data 2020. 432 (Geneva, Switzerland, 2020).

4 Platt, L. et al. Prevalence and burden of HCV co-infection in people living with HIV: a global systematic review and meta-analysis. The Lancet Infectious Diseases16, 797-808, doi:10.1016/s14733099(15)00485-5 (2016).

5 Rashti, R. et al. Global prevalence of HCV and/or HBV coinfections among people who inject drugs and female sex workers who live with HIV/AIDS: a systematic review and meta-analysis. Archives of Virology165, 1947-1958, doi:10.1007/s00705-020-04716-1 (2020).

6 UNAIDS. Fast-track - Ending the AIDS epidemic by 2030, <https://www.unaids.org/en/resources/documents/2014/JC2686_WAD2014report> (2014).

7 UNAIDS/WHO. Guidelines for using HIV testing technologies in surveillance: selection, evaluation and implementation. 42 (Geneva, Switzerland, 2009).

8 Tarasuk, J. et al. National findings from the Tracks survey of people who inject drugs in Canada, Phase 4, 2017-2019. Canada Communicable Disease Report46, 138-148, doi:10.14745/ccdr.v46i05a07 (2020).

9 Cholette, F. et al. Dried blood spot specimens for SARS-CoV-2 antibody testing: A multi-site, multi-assay comparison. Plos One16, doi:10.1371/journal.pone.0261003 (2021).

10 Ly, T. D. et al. Evaluation of the sensitivity and specificity of six HIV combined p24 antigen and antibody assays. Journal of Virological Methods122, 185-194, doi:10.1016/j.jviromet.2004.08.018 (2004).

11 Seignères, B. et al. Multicenter clinical evaluation of the new 3rd generation assay for detection of antibodies against hepatitis C virus on the VIDAS((R)) system. Journal of Clinical Virology78, 20-26, doi:10.1016/j.jcv.2016.03.001 (2016).

12 Barquin, D. et al. HIV-1 diagnosis using dried blood spots from patients in Kinshasa, DRC: a tool to detect misdiagnosis and achieve World Health Organization 2030 targets. International Journal of 
Infectious Diseases111, 253-260, doi:10.1016/j.jij.2021.08.035 (2021).

13 Carrasco, T. et al. HCV diagnosis and sequencing using dried blood spots from patients in Kinshasa (DRC): a tool to achieve WHO 2030 targets. Diagnostics11, 522, doi:10.3390/diagnostics11030522 (2021).

14 Gaballah, A. M. \& Esawy, M. M. Comparison of 2 different antibody assay methods, Elecsys Anti-HCVII (Roche) and Vidas Anti-HCV (Biomerieux), for the detection of antibody to hepatitis C virus in Egypt. Diagnostic Microbiology and Infectious Disease92, 107-111, doi:10.1016/j.diagmicrobio.2018.05.013 (2018).

15 Hyun, J. et al. Evaluation of the VIDAS Anti-HCV assay for detection of hepatitis C virus infection. Annals of Laboratory Medicine36, 550-554, doi:10.3343/alm.2016.36.6.550 (2016).

16 Miedouge, M., Greze, M., Bailly, A. \& Izopet, J. Analytical sensitivity of four HIV combined antigen/antibody assays using the p24 WHO standard. Journal of Clinical Virology50, 57-60, doi:10.1016/j.jcv.2010.09.003 (2011).

17 Salvetti, S., Lavinia, F. \& Montenora, I. Performance study of the automated immunoassay test antihepatitis $\mathrm{C}$ virus VIDAS $\circledast$ for the qualitative detection of antibodies anti-hepatitis $\mathrm{C}$ virus. Microbiologia Medica31, 17-20, doi:10.4081/mm.2016.4808 (2016).

18 Sandstrom, P. \& Meyers, A. Interim guidance on the use of the Abbott ID NOW ${ }^{\mathrm{TM}}$ instrument and COVID19 assay. Canada Communicable Disease Report46, 422-426, doi:10.14745/ccdr.v46i1112a09 (2020).

$19 \mathrm{Wu}, \mathrm{H}$. et al. Hospital capacities and shortages of healthcare resources among US hospitals during the coronavirus disease 2019 (COVID-19) pandemic, National Healthcare Safety Network (NHSN), March 27July 14, 2020. Infection Control \& Hospital Epidemiology, 1-4, doi:10.1017/ice.2021.280 (2021).

20 Sikombe, K. et al. Accurate dried blood spots collection in the community using non-medically trained personnel could support scaling up routine viral load testing in resource limited settings. PLoS One14, e0223573, doi:10.1371/journal.pone.0223573 (2019).

21 van Loo, I. H. M., Dukers-Muijrers, N., Heuts, R., van der Sande, M. A. B. \& Hoebe, C. Screening for HIV, hepatitis $B$ and syphilis on dried blood spots: A promising method to better reach hidden high-risk populations with self-collected sampling. PLoS One12, e0186722, doi:10.1371/journal.pone.0186722 (2017).

22 Biondi, M. J. et al. Hepatitis C core-antigen testing from dried blood spots. Viruses11, doi:10.3390/v11090830 (2019).

23 Villar, L. M. et al. Assessment of dried blood spot samples as a simple method for detection of hepatitis B virus markers. Journal of Medical Virology83, 1522-1529, doi:10.1002/jmv.22138 (2011). 
24 WHO. HIV assays: operational characteristics, HIV rapid diagnostic tests (detection of HIV-1/2 antibodies). 80 (Geneva, Switzerland, 2013).

25 Cheuk, E. et al. Transitions: Novel study methods to understand early HIV risk among adolescent girls and young women in Mombasa, Kenya, and Dnipro, Ukraine. Frontiers in Reproductive Health2, doi:10.3389/frph.2020.00007 (2020).

26 McClarty, L. M. et al. Characterizing HIV risk and vulnerability among commercial sex workers in Zaporizhzhya, Ukraine. країнський соціум [Ukrainian Socium]1, 28-42 (2016).

27 Landis, J. R. \& Koch, G. G. The measurement of observer agreement for categorical data. Biometrics33, 159-174, doi:10.2307/2529310 (1977).

28 Schisterman, E. F., Perkins, N. J., Liu, A. \& Bondell, H. Optimal cut-point and its corresponding Youden Index to discriminate individuals using pooled blood samples. Epidemiology 16, 73-81, doi:10.1097/01.ede.0000147512.81966.ba (2005).

\section{Figures}


(A)
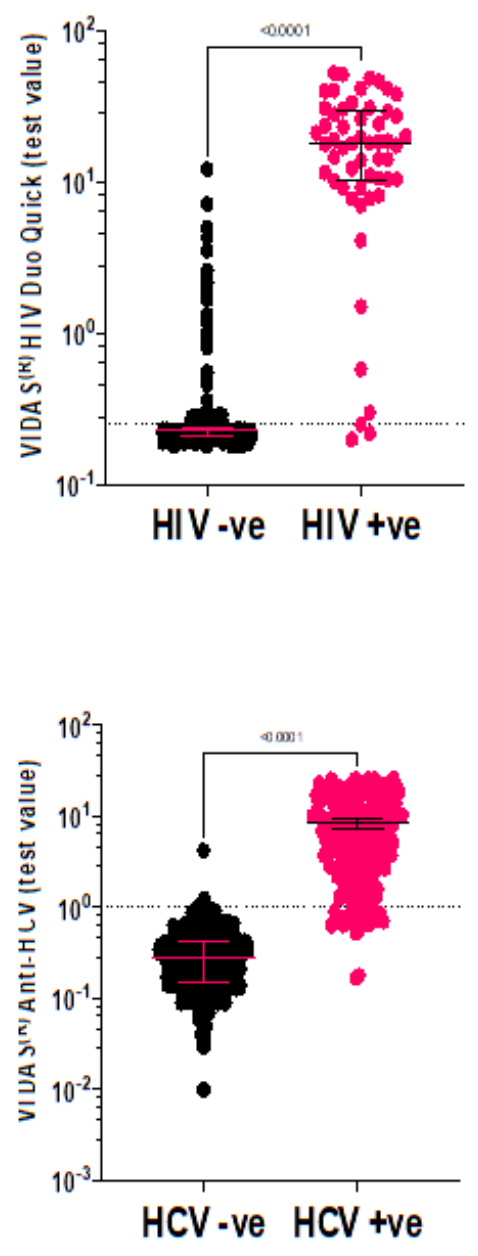

(B)
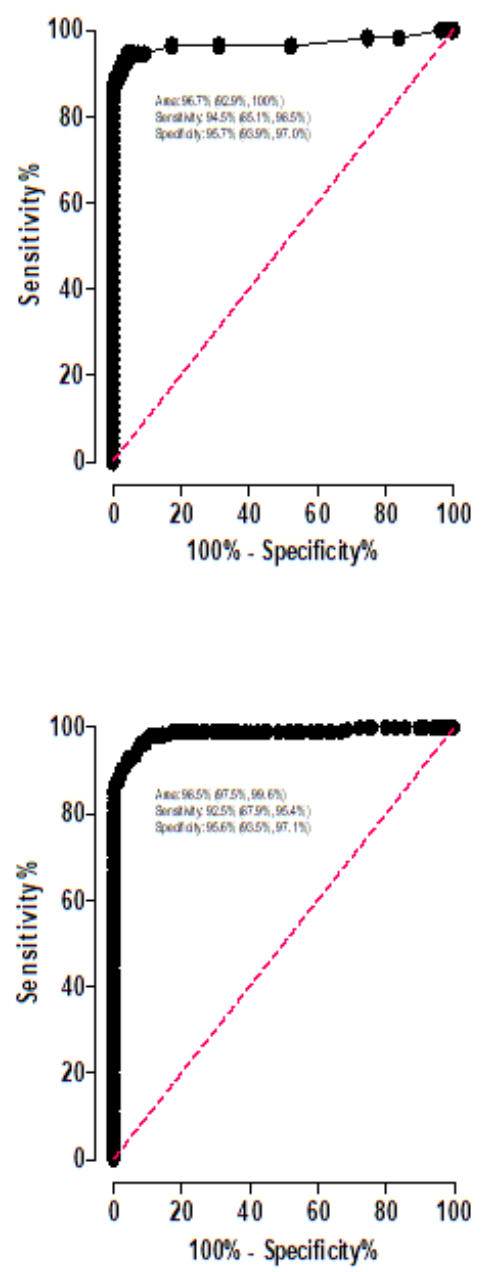

(C)
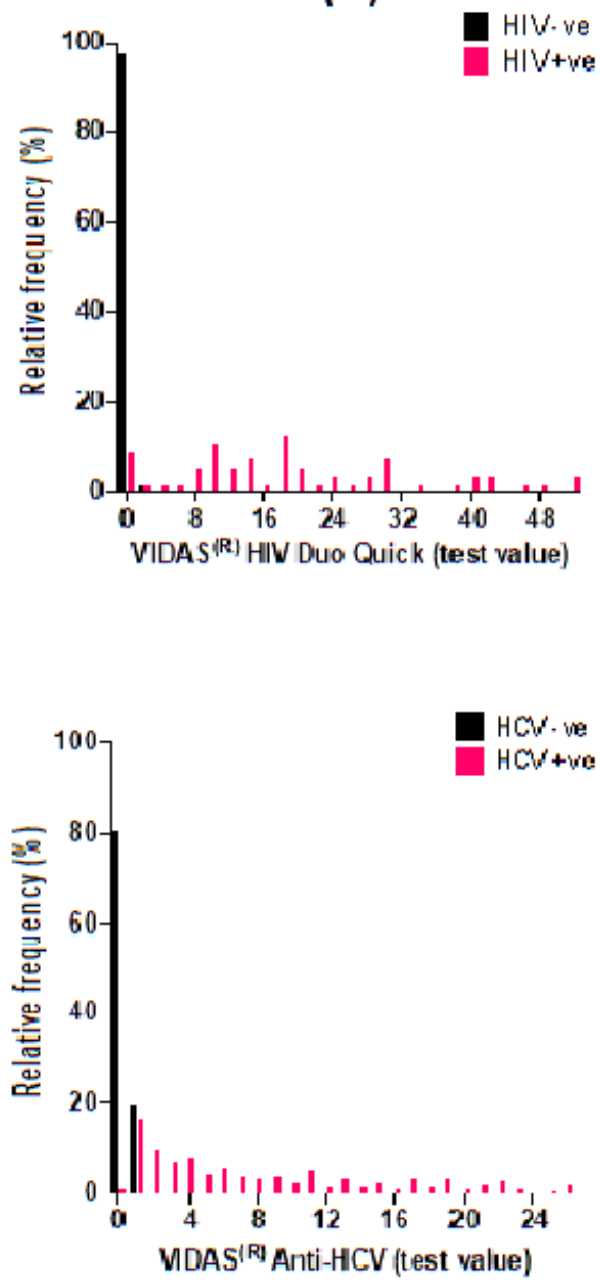

Figure 1

VIDAS® HIV Duo Quick and Anti-HCV test valuesfor DBS samples. (A)Median test values with interquartile ranges (IQR); median test value for HIV -ve DBS: 0.23 (IQR: $0.21,0.24$ ), median test value for HIV +ve DBS: 17.93 (IQR: 10.15, 29.12), median test value for HCV -ve: 0.28 (IQR: 0.15, 0.43), median test value for HCV +ve: 5.99 (IQR: 2.23, 12.70); test value cutoffs recommend by the manufacturer are identified by the dotted lines(B) Receiver operating character is ticcurves $C$ )Distribution of test values.

\section{Supplementary Files}

This is a list of supplementary files associated with this preprint. Click to download.

- Supplementarymaterial.docx 